\title{
Conceptual Paper: Digital Preservation Strategies in Archival Institution
}

\author{
Adila Ismail ${ }^{1, *}$, and Habee Bullah Affandy ${ }^{1}$ \\ ${ }^{1}$ School of Human Development and Technocommunication, Universiti Malaysia Perlis
}

\begin{abstract}
In this paper, the overall overview of concept of preservation regardless if it is preservation of physical records/archives or digital records/archives is discussed. The concept of the preservation discussed includes preventive preservation, restorative preservation, and content preservation. Then the concept of digital preservation. Furthermore, this paper highlighted the strategies and methods used in implementing the preservation of digital records/archives. The issues those are involved in the digital preservation implementation are also discussed.
\end{abstract}

\section{Introduction}

The advancement of technology has given birth to records that exist in digital forms. With the fast rate of technological advancement, more and more records are produced in digital form. Also, the characteristics of digital form records that are easily access further promotes the transition from typical 'physical' documents to digital forms. According to Cumming and Findlay (2010), to ensure that digital records can be reliable and are of value, digital records need to have certain characteristics. Firstly, digital records need to be meaningful and trustworthy to be relied on for business. The second characteristic is that digital records must be fixed, inviolate representations of business activity, preserved in context and protected from loss and alteration.

Electronic records have been used widely in many organizations and the problem arises when it comes to the preservation of electronic records for the purpose of long term access. On a national level in Malaysia, the responsibility of preserving the digital records falls on the National Archive. An archival institution is the facility responsible for preserving records of enduring value. The preservation of records involves both ensuring that the record contains the same information it did when it was archived and ensuring that the record can be viewed using existing technology. It also involves maintaining security and intellectual property rights [1]. Various strategies, policies, and standards have been established by different organization around the world to ensure that the preservation process is effectively implemented. The problem of preserving digital records for long-term access seems straightforward but requires careful consideration about process and technology. Until today there has been no storage platform that can be trusted to store the last existing copy of a critical electronic record. Preserving digital information is more difficult than preserving records on materials such as paper or film. The sheer volume and the volatility introduced by digital demand new software architecture capable of scaling and of preventing accidental changes to the records [1].

Archives preserves a trivial portion of the totality of records and, in the current environment, the digital record they do preserve is not the most important part of that record nor acquired in a manner that ensures its authenticity. Nevertheless, by transferring digital records to archives, archivists become responsible for its preservation which they are, mostly, ill-equipped to provide [2].

Various organizations from foreign countries have designed various strategies in preserving the digital records either on a national level or on an institutional level. Due to the importance of digital records these strategies are seen as initiatives to protect the longevity of the digital records.

Digital records play an important role to support the government functions and its accountability as well as valuable sources of information [3]. For an organization that are responsible for the preservation of digital records on a national level, it is important for the

National Archive of Malaysia to provide and implement a well-designed strategy to ensure that the preservation procedures protect the records existence itself and also its characteristics that ensures the value of the records.

\section{Overview of the Concept of Preservation}

According to MS ISO 223-1 (2009), preservation are processes and operations involved in ensuring the technical and intellectual survival of authentic records 
through time. This refers to preservation is seen as means to protect the records within an organization from any threats that may cause the records to be damaged or destroyed over time. The records within an organization are very important in supporting the ongoing business of an organization. Records are used to solve business problems, evidence to business transactions and also functions as the corporate memory for the organizations. Due to these values, it is important or an organization to protect their records in ensuring that it can be accessed for active use and for long term use (Archives). This is where preservation comes in. The concept of preservation can be categorized in three categories. This includes preventive preservation, restorative preservation and content preservation.

Preventive Preservation: According to the National Library of France (2011), Preventive Preservation refers to the range of direct and indirect actions undertaken on collections and aimed at preventing degradation and prolonging document life spans: carrying out environmental checks, preparing and monitoring an emergency plan, and transferring to alternative media. This statement by the National Library of France, relates to the overall activities in ensuring the physical protection of the materials. The activities involved ensure that the materials are protected from any threats that can put the materials at risk of damages and destructions. Preventive preservation can significantly reduce or even prevent premature deterioration by activities such as environmental control, disaster preparedness, security, storage and handling, reformatting, binding and repair, and conservation treatment.

Restorative Preservation: This concept focuses more on the physical records rather than the digital records. Paper records may not be as sensitive as electronic records, but cautions must be taken in handling and protecting paper records. In doing so, the Public Office of Northern Ireland has developed the Code of Practice for Staff Handling Records to ensure that the records are well handled; such as by not folding and bending the documents as it can crumples the documents; or the staff of the records office must never mark documents with any type of pen or erase anything on them and do not use glue, self-adhesive tape, or correcting fluid anywhere near the documents. Even though the original paper records have stain on it, never used correction fluid to erase as it will affect the originality of the records and might also make it worst. Other than that, the use of selfadhesive tape is prohibited due to the possibility of gluing together unrelated documents; etcetera.

Content Preservation: This concept of preservation regards more with digital records. Digital records are more at risk to content changes rather that paper records. Due to its existence in a digital form, it is at risk of alteration. The content of the digital records must be preserved in order to maintain the authenticity and reliability of electronic records. In content preservation, the need of various hardware and software is never low especially in accumulating the content. The requirements in content preservation are to master the variety of computing technologies to do the routine management such as backup and update [4]. The concept of content preservation described by Han (2004) described the management of digital records based on the usage of various tools. The sensitivity and vulnerability that resides within digital records resulted in the need of detail in the management of the records. The combination of technology such as hardware and software can achieve the main objective of content preservation which is to ensure accessibility of the digital content.

\subsection{The Concept of Digital Preservation}

Digital preservation is defined very broadly and refers to all of the actions required to maintain access to digital materials beyond the limits of media failure or technological change. Those materials may be records created during the day-to-day business of an organization, "born digital" materials created for a specific purpose or the products of digitization projects [5]. Digital preservation consists of the processes aimed at ensuring the continued accessibility of digital materials. To do this involves finding ways to re-present what was originally presented to users by a combination of software and hardware tools acting on data. [6]. To achieve this requires digital objects to be understood and managed at four levels: as physical phenomena; as logical encodings; as conceptual objects that have meaning to humans; and as sets of essential elements that must be preserved in order to offer future users the essence of the object.

According to UNESCO (2011), the most significant threats to digital continuity concern loss of the means of access.

Digital materials cannot be said to be preserved if the means of access have been lost and access becomes impossible. Only with proper access that a digital records can be fully utilized. The purpose of preserving digital materials is to maintain accessibility which is the ability to access their essential, authentic message or purpose. Digital preservation involves choosing and implementing an evolving range of strategies to achieve the kind of accessibility discussed above, addressing the preservation needs of the different layers of digital objects.

\section{The Conceptual Framework}

In implementing digital preservation within an organization, certain strategies must be developed to support and ensure that the entire procedure and processes are well designed and managed. According to the National Archive of Malaysia (2003), the strategy should be developed and implemented organization-wide targeted at identifying, managing, preserving and ensuring continued access to digital records. This statement indicates that the design of the strategy must be on an organizational scale consisting of different 
departments that relates with the preservation process, human resources, financial support and technological infrastructure. In other words, the strategy must include all activities regarding the preservation of electronic records. The strategy must also cover the procedures in ensuring the access towards the digital records after preservation. Without proper access, the records are useless even though they are well preserved.

Based on several literatures, a framework can be developed to describe the digital preservation strategy implementation. The strategy is divided into two stages: i) Initial Stage and ii) Implementation Stage. The framework is shown in Fig. 1.

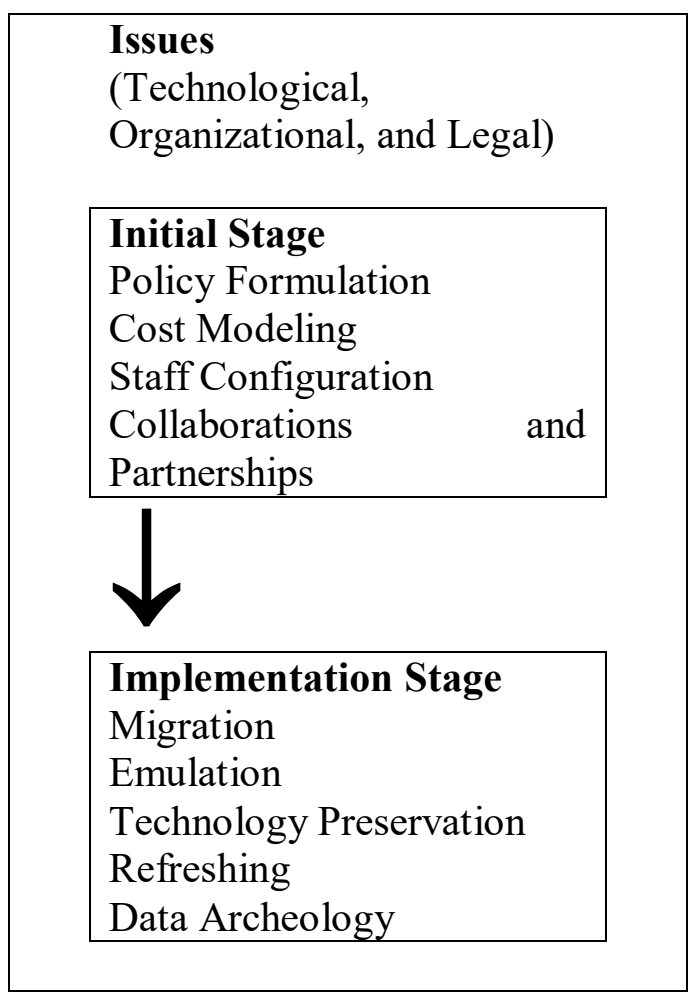

Fig. 1.Digital Preservation Strategy Framework

The framework described 3 major elements in the digital preservation strategy. The first is the initial stage of the strategy. The initial stage refers to works being done before the actual digital preservation processes takes place. The works involved during this stage are policy formulation, cost modeling, staff configuration, and collaborations and partnerships. The next stage in the framework is the Implementation Stage. This refers to the actual digital preservation works. There are five digital preservation techniques that are described in the framework; includes migration, emulation, technology preservation, refreshing and data archeology. The third element in the digital preservation strategy framework is issues. This refers to the issues surrounding the implementation of the digital preservation works. Institutions that are involved in the digital preservation works should address these issues to ensure that the digital preservation works within the institution are well implemented.

\subsection{Initial Stage}

This is the stage within the strategy that establishes a foundation for the implementation. Every process within this stage ensures that the preservation can proceed and sustained.

Policy Formulation: One vital element in the digital preservation strategy is the policy development. The Oxford English Dictionary (OED) puts it more concisely and defines "preservation" as the art of "keeping safe", "keeping alive", "maintaining" and "retaining", while defining a policy simply as: "a course or general plan of action". Therefore, a preservation policy at its most basic is a plan of action for safe keeping. In some of the literature on preservation, the words policy and strategy are often used interchangeably. According to the OED a strategy is "the art of war"; in our case, war against decay and destruction; it is also "the management of an army in a campaign", in other words: the management of all the tools and skills at our disposal to fight against decay. The difference is that a policy, a plan of action, should address the questions of what needs to be preserved, why, for what purpose and for how long, while a strategy addresses the questions of how this should be done and in what order. An effective digital records preservation strategy should incorporate formal policies and procedures governing the agency approach for the maintenance and preservation of digital records. The development of a good public policy requires the appropriate political climate as well as the cooperation of numerous stakeholders [7]. Cloonan and Sannet suggested that in designing the policy, it must be in line with the current politic environment. This is because policies that go against current political environment may cause a misunderstanding among many parties. It is also important that in a digital preservation policy development, an organization should cooperate with various stakeholders in the design process. This is to ensure that the policy is well communicated to the stakeholders and these policies are well accepted. Cloonan and Sannet (2002) indicated that policy will naturally evolve rapidly at the outset of a program when the practitioners encounter new, possibly unanticipated features of the program that require policy decisions.

There are several reasons why digital preservation policies are needed. With ever-dwindling resources and many more claims on those that are available, the need for accountability and the need to justify expenditure have become more pressing. Organizations and institutions, particularly their governors and funders, but also their users, expect a policy as a sign of accountability and as a tangible expression of intent, as well as something that can be monitored [8]. A policy is often required when bidding for funds and to demonstrate that such funds can and will be used responsibly and to good long term effect. Only with adequate funding can a digital preservation procedures be fully implemented. A policy is also required for developing a preservation strategy and to plan coherent preservation programs [8]. With a well-designed 
strategy, it will ensure that the digital preservation processes are well managed.

Cost Modeling: Decisions about preserving information should consider the costs [9]. However, there are no proven techniques for estimating the costs of long-term preservation of digital information. Russell and Weinberger (2000) suggest that the ongoing costs of digital preservation span a more extended timeframe than traditional preservation and will therefore require resource commitments of a different nature. According to Cloonan and Sanett (2002), the strategies in the preservation of electronic records must include the estimation of cost; includes the cost for staff, equipment, space, energy, research and development, and other related costs. These elements are important to ensure that the preservation processes and procedures can be ongoing and be completed in time.

The cost modeling should also include the cost for the access of the materials, cost for long term creation and maintenance of digital materials, production of metadata, supplies, contingency and administration [7]. It is important for digital records to be maintained regularly to ensure its accessibility at present and for future use. The cost of the maintenance is highlighted so that electronic records can be sustained as evidential value. Cloonan and Sannet (2002) also indicated that the cost of staff and equipment consistently need large figures because the cost for staff and equipment will vary over time. New upgraded equipment needs larger cost and with the cost modeling, it will help to estimate the cost needed for the preservation equipments.

Staffing Configuration: A well designed digital records preservation strategy must consider the staffing configuration. In Malaysia, the National Archive of Malaysia advises the public offices to assign responsibility for the management of long term digital records to an appropriate area within an organization [3]. This is to ensure that the management of the digital records within an organization is under the supervision and management of a specific department. This department must consist of professionals with adequate knowledge and skills in records management practice.

Collaborations and Partnerships to Preserve Digital Records: According to the National Archive of Georgia (2007), another element in the digital records preservation strategy is through collaborations and partnerships. This is considered more of a program initiative rather than a procedure. This includes:

a) Identify appropriate partners and stakeholders that are able to contribute to the statewide effort

b) Establish agreements on responsibilities and roles

c) Pursue agreements that provide a reliable basis for ongoing accessibility over time

d) Help identify and develop policies, procedures and tools to support such an aim

e) Work with creators, publishers and re-users of digital content to encourage practices that will enable, rather than hinder, preservation f) Work with government agencies and officials to develop legislative and funding frameworks that will enable cost-effective preservation.

\subsection{Implementation Stage}

After the foundation works have been done during the initial stage, only then can the preservation work itself (dealing with the materials) can be done. This is where the strategy focuses on the implementation stage. The strategy involved the techniques to be used in preserving digital records or archives. Bear in mind that not all technique must be done in the preservation works. Archival institutions should only used techniques that are suitable for them.

There are various digital preservation techniques implemented by organizations in preserving their digital records. Different technique will give different impact to the organization whether it is bad or good. According to Knight (2010), the approach to digital preservation must be related to the provenance, context, authenticity and integrity. The process of digital preservation must not neglect the characteristics of records which are needed to be maintained and preserved. Records are not considered of any value if its authenticity and integrity is abandoned. There are various techniques that include migration, emulation, technology preservation, refreshing and data archeology. Developed countries may not implement all these techniques but only those techniques that are suitable for their organizations.

Migration: The migration of digital information refers to the periodic transfer of digital materials from one hardware and software configuration to another, or from one generation of computer technology to a subsequent generation [10]. The purpose of migration is to preserve the integrity of digital objects and to retain the ability of users to retrieve, display, and use them in the face of constantly changing technology.

There are several countries that implement migration as one of the procedures in their digital records preservation strategy. According to Lee et al. (2002), National Archives and Records Administration or NARA has undergone a project which has been led by San Diego Supercomputer Center which aims to develop a persistent archive to support the storage of archival materials, search for archival materials as well as the preservation of digital collections. The ultimate goal of the project is to preserve not only original data but also the context that permits the data to be interpreted [11]. The National Archive of United Kingdom is seen as one organization that implemented migration in their digital records preservation strategy. Migration according to the National Archives is overcoming technological obsolescence by transferring digital resources from one hardware or software generation to the next. It is seen that migration is done by transferring the old version of software and hardware to the current version to facilitate better access for the digital records. Technology advances in a rapid rate thus it is important that digital records are migrated from old version to newer ones to 
avoid the records being obsolete and unreadable. Also with new technology it will assist in increasing efficiency both in term of process and access.

Another organization that opted for the migration technique is the National Archive of Australia [3]. The National Archive implemented migration when the older formats of documents became obsolete. Based by the definition of the National Archive of Australia, migration is the process of converting a digital object from one data format to another [12]. The authors also indicate that migration is the action done by archivist to preserve digital records. The action involved the change of format of the content of the records from obsolete format to the current format. This method is mainly done to ensure the accessibility of those records before it becomes obsolete and unreadable.

Based on the migration technique implemented by three different organizations, all organization shares the same intent in choosing migration technique in their digital preservation strategy which is to ensure accessibility of the digital records.

Emulation: According to the National Archive of United Kingdom, emulation refers to a technique in overcoming technological obsolescence of hardware and software by developing techniques for imitating obsolete system on future generations of computers. The authors indicated that in order to preserve electronic records, a replica of the old system should be developed. The author view of emulation is reproducing the same systems or technologies for digital records activities by using the current generations of technologies. The author argued that it is important to ensure that the whole content, context and structure of the digital records will never change although emulation has been applied in the digital preservation works. The National Archive recommended that emulation camp must be done for active file. This is mainly because active files have been used for many purposes thus it crucial to emulate those files because it will affect many activities in the National Archive with regards to the digital records. Emulator programs can be designed and run on future computer platforms. The emulator is programmed to mimic the behavior of old hardware platforms and operating system software, such as for games and executable files [13. However, this strategy does not involve preserving ageing hardware and original operating system software. The goal of emulation is to preserve the look and feel of the digital object as well as its functionality. The essence of this strategy is to copy the technical context of the resource allowing the original object or a refreshed copy of the original object to be used in the future [11]. This is in line with the statement by Heslob, Davis and Wilson (2002), where they indicated that the emulation process always maintains the exact look of the records that need to be preserved or in other words the originality of the structure of the records. This is why the National Archive of Australia opted for this technique due to its characteristic that preserved all aspects of the digital records.
Technology Preservation: Technology preservation is based on preserving the technical environment that runs the system, including software, media drives and so on [14]. This solution supposes that complete museums of obsolete equipment could be maintained in order to replicate any old configuration of hardware and software. This strategy involves preserving an original application program, operating system software, and hardware platform [11]. The advocates of this strategy emphasize that the original environment needs to be run to really preserve the behavior as well as the look and feel of the digital object. For some digital objects, this may be the best solution at least in the short-term because it ensures that the material is accessible by preserving the access tools as well as the object itself [11].

However, various issues including space, maintenance, and costs may make this impossible in the longer term. Specifically, equipment ages and breaks, documentation disappears, vendor support vanishes, and the storage medium as well as the equipment deteriorates [11]. This strategy also limits the portability of the resource since it will depend on hardware stored in specific locations [14]. Technology preservation is more of a disaster recovery strategy for use on digital objects that have not been subject to proper digital preservation strategy. It offers the potential of coping with media obsolescence, assuming the media has not decayed beyond readability [14].

Refreshing: The physical media that stores the digital records can deteriorate in time. The condition of the physical media might not be the same as when it is created because of degradation. In order to overcome the eventual degradation of the storage media, there will generally be some point at which the information contained in these carriers will have to be duplicated or reconstructed on another instance of medium, or a new medium all together [15]. According to the Cornell University Library (2007), refreshing involved copying content from one storage medium to another and its targets only media obsolescence and is not a full-service preservation strategy. This refers to the movement of the digital content from an obsolete physical medium to the new medium. The objective of this technique is to cope with technological changes that can cause digital media storage becoming obsolete. Obsolete physical storage might affect the readability of the digital content.

Data Archeology: Digital or Data Archeology includes methods and procedures to rescue content from damaged media or from obsolete or damaged hardware and software environments [14]. Cornell University Library emphasized that digital archeology is related to the preservation of the data when there is possibility of the tools in managing the electronic records become obsolete. The data will be preserved in archive which contains all information about the records. Data archives ensure long term availability of data through standardizing formats, migrating formats as technologies change, and guarding against general degradations in the data [16]. The authors stressed that the availability of 
data is dependent on technology. Technology which involves the tools in data archeology can affect the accessibility of the data especially for long term preservation. The data that resides in electronic records might achieve long term preservation if the changes in technology can be overcome and solve. On the other hand, data archives can provide a third party system for ensuring the integrity and accessibility of research data for the future [16]. The authors indicated that data archive can help data archeology in terms of maintaining the integrity of the original data which means the original characteristic of the data.

According to the National Archive of Malaysia (2003), there are five factors that any agencies and organizations need to consider before implementing certain digital preservation technique. These include cost implementation, technical complexity, technology compatibility, impact on business operations and overall effectiveness of the approach. Cost implementation is one factor in choosing the suitable methods of digital preservation, as it is quite expensive to implement. Due to this, it is not advisable for archival institutions to implement all methods. The process of choosing the right method is vital for an archival institution.

\subsection{Issues in Digital Preservation}

Another element in the digital preservation framework is issues. Not just the framework concern with the works involved but also it concern with the issues surrounding the digital preservation works. It is important for archival institutions to analyze the issues that exist within the scope of digital preservation. Digital preservation requires considerations from all aspects on all level of implementation from the initial planning, the implementations of the processes and after the completion of the processes. Several issues are recognized regarding the preservation of digital records, and are categorized into three major categories which are Technological Issues, Organizational Issues and Legal Issues.

\section{a) Technological Issues}

Digital Media: Digital materials are especially vulnerable to loss and destruction because they are stored on fragile magnetic and optical media that deteriorate rapidly and that can fail suddenly from exposure to heat, humidity, airborne contaminants, or faulty reading and writing devices [17]. Digital media are subject to destruction and deterioration in new ways, though unintended loss can be avoided if procedures are adapted to the needs of the technology. Precautions can be taken which will help significantly to reduce the danger of loss and include: Storing in a stable, controlled environment, implementing regular refreshment cycles to copy onto newer media, making preservation copies (assuming licensing/copyright permission), implementing appropriate handling procedures and transferring to "standard" storage media [5].
Changes in Technology: Unlike the situation that applies to books, digital archiving requires relatively frequent investments to overcome rapid obsolescence introduced by galloping technological change [18]. Because digital material is machine dependent, it is not possible to access the information unless there is appropriate hardware, and associated software which will make it intelligible. The certainty that there will be frequent technological change poses a major challenge. Precautions can, and should be taken, which will greatly reduce the risk of inadvertently losing access to a resource because of changes in technology [5].

Authenticity and Context: The approach to digital preservation of digital records must be related to the provenance, context, authenticity and integrity [19]. This means that the electronic records must maintain its original condition without alteration after the preservation process. The authenticity and integrity of electronic records are mutual concern [7] because records cannot be considered as records if the records do not maintain its authenticity and integrity.

b) Organizational Issues

Costs: The cost of digital preservation cannot be easily isolated from other organizational expenses, nor should it be. Digital preservation is essentially about preserving access over time and therefore the costs for all parts of the digital life cycle are relevant. In that context even the costs of creating digital materials are integral in so far as they need to include cost elements which will ultimately facilitate their long-term preservation [5]. Nonetheless the exercise of calculating costs, however complex, is a valuable and necessary task to establish cost-effective practices and a reliable business model. The cost of the labor required for digital preservation will be the most significant by far and includes not only dedicated experts but varying proportions of many staff such as administration, management, IT support, legal advisers etc [5].

Expertise: The need for digital preservation expertise is high: asked to rate staff as expert, intermediate, or novice, only 8 of the 54 institutions considered their staff at the expert level. [17]. The dramatic speed of technological change means that few organizations have been able even fully to articulate what their needs are in this area, much less employ or develop staff with appropriate skills. In addition, there is little in the way of appropriate training and "learning by doing" can often be the most practical interim measure [5].

Selection: The enormous quantity of information being produced digitally, its variable quality, and the resource constraints on those taking responsibility to preserve long-term access, makes selectivity inevitable if the objective is to preserve ongoing access. In the digital environment, it is possible to by-pass the traditional distribution channels, as well as filtering and quality control processes. While there are benefits for users in terms of swift access, there are also difficulties in terms of quality control. Selecting quality materials for long- 
term retention therefore places a burden on organizations in terms of resources and also in terms of the potential impact of selection [5]. In cases where there may be multiple versions, decisions must be made in selecting which version is the best one for preservation, or whether more than one should be selected. Sampling dynamic resources as opposed to attempting to save each change, may be the only practical option but may have severe repercussions if the sampling is not undertaken within a well-defined framework and with due regard to the anticipated contemporary and future needs of the users [5].

\section{c) Legal Issues}

Intellectual property rights (IPR) and preservation: Copyright and other intellectual property rights (IPR) such as moral rights have a substantial impact on digital preservation. The preservation of digital materials is dependent on a range of strategies, which has implications for IPR in those materials. The IPR issues in digital materials are arguably more complex and significant than for traditional media and if not addressed can impede or even prevent preservation activities. Consideration may need to be given not only to content but to any associated software. Simply copying (refreshing) digital materials onto another medium, encapsulating content and software for emulation, or migrating content to new hardware and software, all involve activities which can infringe IPR unless statutory exemptions exist or specific permissions have been obtained from rights holders [5].

Privacy and Confidentiality: Information held within the repository may be subject to the Data Protection Act or similar privacy legislation protecting information held on individuals. Information may also be subject to confidentiality agreements. Privacy and confidentiality concerns may impact on how digital materials can be managed within the repository or by third parties, and made accessible for use [5].

\section{Conclusion}

MS ISO 223 was a standard regarding records management that was established to assist Malaysia in becoming a structured country. According to MS ISO 223-2 (2009), preservation strategies for records especially electronic (digital) records may be selected on the basis of their ability to maintain the accessibility, integrity and authenticity of the records over time, as well as cost effectiveness. The nature of the electronic (digital) records must be maintained even though the records had undergone preservation activities such as copying, conversion and migration.

The National Archive of Malaysia has indicated the importance of the digital records in supporting the government functions but there are no publications or researches that describe in detail the strategies being done by the National Archive of Malaysia (as it is the institution that is responsible in the digital preservation on a National basis). Without detailed research, it is difficult to ensure that the strategies being implemented are effective and suitable or not.

\section{References}

1. A. Rodriguez, Computer Technology Review, 25(3), 17-19 (2005)

2. D. Bearman, Addressing selection and digital preservation as systemic problems. International Conference organized by Netherlands National Commission for UNESCO Koninklijke Bibliotheek (National Library of the Netherlands). The Hague, 45 November 2005.

3. National Archive of Malaysia. (2003). Electronic records management and archives management policy. Kuala Lumpur: National Archive of Malaysia.

4. Y. Han, Digital content management: The research for a content management system. Library Hi Tech. 22 (4), 355-356 (2004)

5. Digital Preservation Coalition. (2008). Digital Preservation Definition and Concepts. Retrieved from www.dponline.org.

6. UNESCO. Concept of Digital Preservation. (2011). Retrieved from www.unesco.org.

7. M. V. Cloonan, S. Sanett, The American Archivist. 65, 70-106 (2002)

8. M. Foot, (2001): Building Blocks for a Preservation Policy. NPO Preservation Guidance Preservation Management Series. 1-13. Retrieved from http://www.bl.uk/services/preservation/npo8.pdf

9. T. Hendley, (1998). Comparison of Methods \& Costs of Digital Preservation. British Library Research and Innovation Report. Retrieved from http://www.ukoln.ac.uk/services/elib/papers/tavistock hendley/hendley.html

10. J. Garrett, D. Waters, Preserving Digital Information, Report of the Task Force on Archiving of Digital Information. The Commission on Preservation and Access and The Research Libraries Group. Washington DC and Mountain View CA (1996)

11. K. Lee, O. Slattery, R. Lu, X. Tang, V. Mc Crary, Journal of Research of the National Institute of Standards and Technology 107 (1), 93-106 (2002)

12. H. Heslob, S. Davis, A. Wilson, (2002). An approach to the preservation of digital records. National Archives of Australia. Retrieved from: http://www. Naa.gov.au/images/an-approachgreen-paper_tcm2-888.pdf.

13. S. Granger (2000). Emulation as a Digital Preservation Strategy. D-Lib Mag. 6(10). Retrieved from

http://www.dlib.org/dlib/october00/granger/10gran ger.html

14. Cornell University Library. (2007). Digital preservation. Retrieved from: http://www.library.cornell.edu/preservation/tutorial /preservation/preservation-03.html.

15. N. Pozo, A. S. Long, D. Pearson, D. (2010). Land of the lost: a discussion of what can be preserved through digital preservation. Library $\mathrm{Hi}$ Tech, 28(2): 290-300 (2010) 
16. J. J. Albright, J. A. Lyle, Political Science and Politics 43(1), 17-21(2010)

17. M. Hedstrom, S. Montgomery, (1998). Digital preservation needs and requirements in RGL member institutions. United States of America: Research Libraries Group. Retrieved from http://www.oclc.org/research/activities/past/rlg/dig presneeds/digpres.pdf.

18. M. Feeney, (1999). Digital Culture: Maximising the Nation's Investment.

19. S. Knight, S. (2010). Early learnings from the National Library of New Zealand's National Digital Heritage Archive project. Electronic Library and Information Systems. 44 (2): 85-97 (2010) 